\title{
A High Performance Image Authentication Algorithm on GPU with CUDA
}

\author{
Caiwei Lin \\ School of Computer Science and Technology, Soochow University, Suzhou, China \\ Email: lincwei@gmail.com \\ Lei Zhao and Jiwen Yang \\ School of Computer Science and Technology, Soochow University, Suzhou, China \\ Email: \{zhaol,jwyang\}@ suda.edu.cn
}

\begin{abstract}
There has been large amounts of research on image authentication method. $M$ any of the schemes perform well in verification results; however, most of them are timeconsuming in traditional serial manners. And improving the efficiency of authentication process has become one of the challenges in image authentication field today. In the future, it's a trend that authentication system with the properties of high performance, real-time, flexible and ease for development. In this paper, we present a CUDA-based implementation of an image authentication algorithm with NVIDIA's Tesla C1060 GPU devices. Comparing with the original implementation on CPU, our CUDA-based implementation works 20x-50x faster with single GPU device. And experiment shows that, by using two GPUs, the performance gains can be further improved around 1.2 times in contras to single GPU.
\end{abstract}

Index Terms-GPU, CUDA, image authentication, semifragile watermarking

\section{INTRODUCTION}

Digital images are widely distributed today over the internet and through other mediums, and there are powerful digital image processing tools which have made perfect image duplication a trivial procedure. Therefore, copyright protection and integrity verification of digital image have become an urgent issue in the digital world. Image authentication technology is important in various types of images' protection. A vast research has been conducted on methods of image authentication, and many useful concepts such as signal transform (DCT, DWT, etc.) and HVS (Human Visual System), was employed to improve the performance and to satisfy the practical requirement. As a result, lots of the algorithms proposed in literatures are complicated in computation, which restrain their being adopted in some real-time occasions. Improving the efficiency of image authentication process has become one of the challenges in this field.

Some researchers have used hardware like FPGA and custom IC as co-processor in image processing, but the procedure of hardware designing and programming is not

Corresponding Author: Lei Zhao a friendly work. On the other hand, nowadays, GPU devices are wildly equipped in personal computers and have a great potential in mass parallel computing capability. Traditional GPGPU (General Purposes Graphical Processing Unit) methods, however, require programmers equipped with graphic knowledge and API, which make the work of designing of GPU parallel computing tedious and hard to debugging. As the release of NVIDIA's Compute Unified Device Architecture (CUDA), researchers can design programs for both CPU and GPU conveniently with a C-like programming language, without knowing fundamental knowledge on computer graphics.

In this paper, we firstly propose an image authentication scheme, and then discuss the implementation of it on CUDA-enabled GPUs, which have been widely equipped in today's PCs. Experimental results show that our CUDA-based method for image authentication gains a good performance in both authentication results and operation speedup. The rest of this paper is organized as follows. Related work is discussed in Section II. In Section III, the hardware model and programming model of CUDA is reviewed. And the image authentication algorithm proposed in this paper is presented in section IV. Section V describes the detail of CUDA-based implementation of the proposed algorithm, and experimental results of the implementation are presented in Section VI. Conclusions are drawn in Section VII.

\section{RELATED WORK}

Generally speaking, image authentication techniques can basically be categorized into two types: digital signature and digital watermarking [1]. Extensive research on image authentication algorithms have been proposed in the last decades, especially on semi-fragile watermarking. Semi-fragile watermarking technology is mainly used for image content authentication. It is required that the authentication method is able to tolerate incidental manipulations while being sensitive to malicious distortions.

Chen proposed an image authentication scheme combines digital signature and watermarking [2], which extracts signature from the original image and embeds 
them back into the image as watermark and avoid additional signature bandwidth for transmission, the algorithm shows not only tolerate JPEG compression but also able to locate the illegal modifications. Ho and Li [3] proposed a semi-fragile watermarking scheme which tolerates JPEG compression and equipped with the properties of nondeterministic block-wise dependence, obliviousness and localize attacks. Lin and Su present a semi-fragile method by employ a new concept of the lowest authenticable JPEG quality (LAJQ), and also prove the validity of pre-compression method mentioned in literatures [3-5]. The scheme in [10] took HVS into account to against special attack of watermarked images. Other schemes can not only localize the modifications, but also recover the tampered region [11].

Although these methods performed well in image authentication, most of them need computational intensive operations, and thus are unable to generate a result instantaneously. Several hardware assisted methods for digital signature and watermarking acceleration have been studied in recent years, for instance, some attempts for accelerating watermarking methods by using dedicated special-purpose hardware like digital signal processor (DSP) and field programmable gate array (FPGA) [6], for example, [14] proposed robust and fragile watermarking acceleration schemes by using custom IC hardware, and Seo and Kim presented a FPGA co-processed method for robust watermarking in DWT domain [15]. Nevertheless, these methods are designed at the hardware level that limit the capability of programming, thus designing different schemes may require complete changes of hardware architectures. What's more, these devices are not commonly available in consumer level PC, which constraints the deployment of related technology. Graphics Processor Unit (GPU) is one of high performance computing platforms and wildly available in today's PC. Brunton and Zhao [7] used GPU as a co-processor, and proposed a watermarking-based content authentication method for real-time video application, and the system was shown to have good performance both on processing efficiency and authentication results. Mohanty [8] presented an efficient, real time and low cost watermarking system for image, by implementing a dedicated processor chip as a coprocessor for the GPU. However, their methods are still focused on hardware designing or required knowledge on graphics API or dedicated processor chips. On the other hand, the new release of GPU programming platform CUDA [9] offers highly parallel computation and flexible programmable environment. Many recent papers have devoted to exploiting the massive parallel computing processors on GPU's massive cores, and lots of problems in the fields of linear algebra [16], image processing [17], biomedical information [18], signal processing [19], etc., have benefited from its speed and parallel processing capability. However, seldom CUDA-based accelerating watermarking or image authentication methods have been presented in literatures. The goal of using CUDA in image authentication field is to establish a high performance, real time, flexible and ease developed system of authentication.

\section{CUDA OVERVIEW}

Compute Unified Device Architecture (CUDA) is a new hardware and software architecture for designing and dealing with computations on the GPU, without the need of mapping them to a graphics API, and allow direct access to GPU processors and device memory. Its straightforward extension of the $\mathrm{C}$ programming style and native interface to GPU's data-parallel model have made the implementation of GPU-based parallel computation a friendly work [9]. However, to achieve high performance, software designers need to acquire knowledge about CUDA's architecture and programming optimize strategy.

\section{A. Hardware Model}

The CUDA device is equipped with a set of SIMD stream multi-processors (SM), and each SM contains several stream processors (SP). For example, NVIDIA Tesla C1060 GPU is build with 240 SP cores, organized in $30 \mathrm{SM}$ streaming multiprocessors. GPU's massive process cores make it naturally to run great amount of concurrent threads at the same time. As a result, GPU is suitable for data-parallel and computing intensive tasks.

CUDA architecture provides access to several types of memory. Global (device) memory can be read and written by all threads. For each thread block, there is a shared memory (on-chip memory) available for all threads within the block, and registers are local storage for each SP. Share memory has low access latency and high bandwidth, similar to an L1 cache. Thus, the access speed of shared memory and register are much faster than global memory, so it is an important strategy for accelerating data access. There are also two additional read-only memory spaces accessible for all threads: the constant and texture memory spaces. Texture memory is a special case of device memory which is cached for locality. Constant memory is cached memory that can be written by the CPU and read by the GPU. To achieve highest throughput, consecutive memory locations must be simultaneously accessed by the threads, which is called memory access coalescing. By coalescing the global memory reads and writes, and avoiding on-chip shared memory bank conflicts, performance gains can be further increased. This is an effective technique for hiding memory latency and will be taken into account in our later implementation.

\section{B. Programming M odel}

Under the CUDA programming model, applications are divided into grain independent tasks. These tasks are parallelized by scalar execution units called threads. A set of threads are organized as thread blocks, and each thread and block is given a unique ID that can be accessed within the thread during its execution. The CPU invokes the GPU by calling a CUDA kernel. In fact, kernel is a special $\mathrm{C}$ function. In a given execution of CUDA kernel, each thread can perform the kernel task on different set of data using the thread and block IDs. The set of all blocks 
run during the execution of a CUDA-kernel is called a grid.

Threads within a block can share data with each other. Besides, it's able to place synchronization points to control the execution flow of all the threads within a block. Threads from different blocks in the same grid can coordinate only via operations in a shared global memory space visible to all threads. This provides fine-grained data parallelism and thread parallelism. 16 consecutive threads in a block form a half-warp, and 2 half-warps form a warp. A warp size of consecutive threads which take the same execution path will be activated at the same clock cycle of GPU. Thus, threads in a warp that execute different task paths (also called diverge) must wait in turn for the next executing clock cycle. Therefore, it is highly suggest avoiding divergence in a warp to achieve substantial efficiencies of processors.

\section{IMAGE AUTHENTICATION ALGORITHM}

In this section, we propose a semi-fragile watermarking algorithm for image authentication. The algorithm extracted features from low and middle frequency domain of DCT coefficient blocks and embedded them into high frequency region.

\section{A. Review of DCT}

Discrete Cosine Transform (DCT) is a transformation function which transforms the representation of data from space domain to frequency domain. DCT method is well known in image processing for it's used in the standard of JPEG compression. As for matrix $\left.\right|_{M \times N}, 2$-dimensional DCT formulate is defined as follow:

$C(u, v)=\partial(u) \partial(v) \sum_{i=0}^{M-1} \sum_{j=0}^{N-1} I(i, j) \cos \left[\frac{\pi(2 i+1) u}{2 M}\right] \cos \left[\frac{\pi(2 j+1) v}{2 N}\right]$

And the corresponding inverse DCT formulate is:

$$
I(i, j)=\sum_{u=0}^{M-1} \sum_{v=0}^{N-1} \partial(u) \partial(v) C(u, v) \cos \left[\frac{\pi(2 i+1) u}{2 M}\right] \cos \left[\frac{\pi(2 j+1) v}{2 N}\right]
$$

Where

$$
\partial(u)=\left\{\begin{array}{l}
\sqrt{\frac{1}{M}}, u=0 \\
\sqrt{\frac{2}{M}}, u \neq 0
\end{array}, \partial(v)=\left\{\begin{array}{l}
\sqrt{\frac{1}{N}}, v=0 \\
\sqrt{\frac{2}{N}}, v \neq 0
\end{array}, 0 \leq u \leq M-1,0 \leq v \leq N-1\right.\right.
$$

\section{B. F eature extraction and embedding scheme}

The steps of image feature extraction and embedding are expressed as follows:

\section{1) Performing block-based DCT}

Divided the original $\mathrm{M} \times \mathrm{N}$ image I into nonoverlapped $8 \times 8$ blocks, where i, j are locations of the blocks in image I, and apply 2D-DCT to each block, then we get DCT-coefficient blocks $C_{i, j}$ (suppose the whole block-wise DCT-coefficient matrix is $\mathrm{C}$ ). For each $8 \times 8$ DCT-coefficient block, the well known zigzag order is employed, and each block is partitioned into low (L), middle (M), high $(\mathrm{H})$ frequency domain, as illustrated in Fig.1.

2) Secure serial-numbers generation

To increase the security of the algorithm and avoid block-wise independence, we employ the Logistic chaotic model and generate a unique serial-number $T(i, j, \mu)$ for each block $C_{i, j}$ using the following equation:

$$
\mathrm{x}_{\mathrm{n}+1}=\mu \mathrm{x}_{\mathrm{n}}\left(1-\mathrm{x}_{\mathrm{n}}\right)
$$

where $\mu(3.5699<\mu<4)$ is an initial private key.
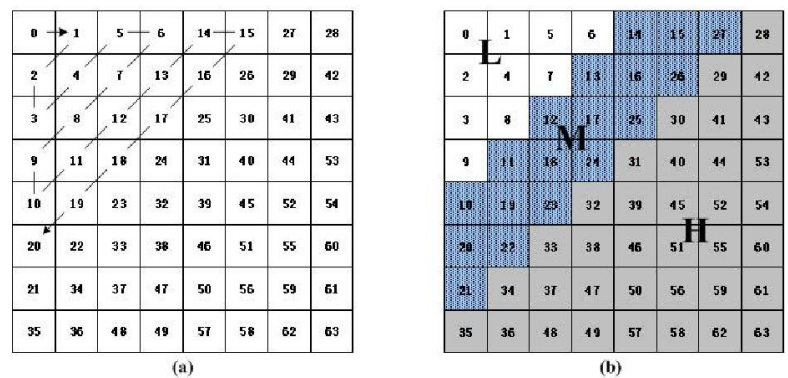

Figure 1. (a) Zigzag order of $8 \times 8$ DCT-coefficient block; (b) Frequency domains $8 \times 8$ DCT-coefficient block.

\section{3) Watermark extraction}

The watermark sequences extracted from image are invariant relationship between two DCT-coefficients in $8 \times 8$ block pairs, which was proved to be robust before and after JPEG compression by Lin and Chang [5] Thus, we will employ this property to extract image features. In order to make later expression clearer, let us take the neighboring block $\mathrm{C}_{0,0}$ and $\mathrm{C}_{0,1}$ for example, and we define the following symbols:

- $\mathrm{K}_{\mathrm{l}}, \mathrm{K}_{\mathrm{m}}$ : tow dynamic secret keys used to pick the positions from the low and middle frequency domain, and they are defined as below:

$$
\begin{aligned}
& \mathrm{K}_{\mathrm{l}}=\lambda_{\mathrm{l}} \oplus \mathrm{T}(0,0, \mu) \oplus \mathrm{T}(0,1, \mu) \\
& \mathrm{K}_{\mathrm{m}}=\lambda_{\mathrm{m}} \oplus \mathrm{T}(0,0, \mu) \oplus \mathrm{T}(0,1, \mu)
\end{aligned}
$$

where $\lambda_{\mathrm{l}}$ and $\lambda_{\mathrm{m}}$ are another two private keys, and $\mathrm{T}(\mathrm{i}, \mathrm{j}, \mu)$ is a serial-number generated by (3).

- $\mathrm{I}_{\mathrm{s}}$ : the zigzag order indexes of four coefficients picked from the low frequency domain by $K_{\text {I }}$, $\mathrm{S}=\{1,2,3,4\}$

- $\mathrm{m}_{\mathrm{s}}$ : another four indexes picked from the middle frequency domain with private key $\mathrm{K}_{\mathrm{m}}$;

Then the low frequency feature vectors $W_{L}{ }_{L}^{1}$ and $W_{L}{ }^{2}$ are extracted using the following rules:

$$
\begin{gathered}
W_{L}^{1}(s)= \begin{cases}1 & C_{0,0}\left(I_{s}\right) \leq C_{0,1}\left(I_{s}\right) \\
0 & \text { otherwise }\end{cases} \\
W_{L}^{2}(s)= \begin{cases}1 & \text { sign }\left(C_{0,0}\left(l_{s}\right)\right) \neq \operatorname{sign}\left(C_{0,1}\left(I_{s}\right)\right) \\
0 & \text { otherwise }\end{cases}
\end{gathered}
$$

and the watermark vector generated from low frequency domain can be defined as:

$$
W_{L}=W_{L}^{1} \oplus W_{L}^{2}
$$

Similarly, for the middle frequency domain, we have: 


$$
\begin{gathered}
W_{M}^{1}(s)= \begin{cases}1 & C_{0,0}\left(m_{s}\right) \leq C_{0,1}\left(m_{s}\right) \\
0 & \text { otherwise }\end{cases} \\
W_{M}^{2}(s)= \begin{cases}1 & \operatorname{sign}\left(C_{0,0}\left(m_{s}\right)\right) \neq \operatorname{sign}\left(C_{0,1}\left(m_{s}\right)\right) \\
0 & \text { otherwise }\end{cases}
\end{gathered}
$$

And define the watermark vector of middle frequency domain as follow:

$$
W_{M}=W_{M}^{1} \oplus W_{M}^{2}
$$

As a consequence, we completed watermark extraction from block pair: $C_{0,0}$ and $C_{0,1}$. The similarly processing will be done for the other block pairs.

4) E mbedding watermark

The step of embedding watermark, we take the partial high frequency domain as watermark embedding region Em. Fig. 2 (a) shows the embedding region of each $8 \times 8$ block. It is in order to ensure the perceptible quality of the image. By pre-quantize the DCT coefficient with a predetermined quality factor [4] [5], the watermarked image will tolerate any number of further compressions of smaller quantization steps. Here we take Q / 4 as the quantization table whose elements are corresponding values equal to $1 / 4$ times of the values from JPEG quantization table $Q$, which is illustrated in Fig.2 (b).

\begin{tabular}{|c|c|c|c|c|c|c|c|}
\hline 0 & 1 & 5 & 6 & 14 & 15 & 21 & 28 \\
\hline 2 & 4 & 7 & 13 & 16 & 26 & 29 & 42 \\
\hline 3 & 8 & 12 & 11 & 25 & 30 & 41 & 43 \\
\hline 9 & 11 & 18 & 24 & 31 & 40 & 44 & 53 \\
\hline 10 & 19 & 23 & 32 & 39 & 45 & 52 & 54 \\
\hline 20 & 22 & 33 & 38 & 46 & 51 & 55 & 60 \\
\hline 21 & 34 & 37 & 47 & 51 & 56 & 59 & 61 \\
\hline 35 & 36 & 48 & 49 & 57 & 58 & 62 & 63 \\
\hline
\end{tabular}

\begin{tabular}{|l|l|l|l|l|l|l|l|}
\hline 15 & 11 & 10 & 16 & 24 & 40 & 51 & 61 \\
\hline 12 & 12 & 14 & 19 & 26 & 58 & 69 & 55 \\
\hline 14 & 13 & 16 & 24 & 01 & 57 & 69 & 56 \\
\hline 14 & 17 & 22 & 29 & 51 & 87 & 80 & 62 \\
\hline 18 & 22 & 37 & 56 & 68 & 109 & 103 & 71 \\
\hline 24 & 35 & 55 & 64 & 81 & 104 & 113 & 92 \\
\hline 49 & 64 & 78 & 87 & 103 & 121 & 1211 & 101 \\
\hline 79 & 92 & 95 & 98 & 112 & 100 & 103 & 99 \\
\hline
\end{tabular}

Figure 2. (a) The region for embedding watermark; (b) JPEG quantization table $\mathrm{Q}$

Still, take $C_{0,0}$ and $C_{0,1}$ for example, four coefficients will be picked out from Em region in each block with a private key $\mathrm{K}_{\mathrm{h}}$, which can be got by the ruler below:

$$
\mathrm{K}_{\mathrm{h}}(\mathrm{i}, \mathrm{j})=\lambda_{\mathrm{h}} \oplus \mathrm{T}(\mathrm{i}, \mathrm{j}, \mu)
$$

where $\lambda_{h}$ here is also a private key. Let $h_{s}^{1}, h_{s}^{2}$ means the indexes of the four embedded coefficients in $C_{0,0}$ and $C_{0,1}$. And $C_{0,0}\left(h_{1}^{1}\right)$ and $C_{0,0}\left(h_{2}^{1}\right)$ will be embed the first two elements of the watermark vector $V_{L}$, and $C_{0,0}\left(h_{3}^{1}\right), C_{0,0}\left(h_{4}^{1}\right)$ will be embedded the first two elements of the watermark vector $W_{M}$. Another two elements of $\mathscr{W}_{L}$ and $\mathscr{W}_{M}$ will be embedded into $C_{0,1}\left(h_{s}^{2}\right)(S=1 \ldots 4)$ respectively. Then, we define the following symbols:

$$
\begin{gathered}
R C_{i, j}\left(h_{s}^{k}\right)=\operatorname{round}\left(C_{i, j}\left(h_{s}^{k}\right) / Q\left(h_{s}^{k}\right)\right) \\
U R C_{i, j}\left(h_{s}^{k}\right)=C_{i, j}\left(h_{s}^{k}\right) / Q\left(h_{s}^{k}\right)
\end{gathered}
$$

$$
\operatorname{Sgn}= \begin{cases}1 & \text { if }\left(U R C_{i, j}\left(h_{s}^{k}\right)-R C_{i, j}\left(h_{s}^{k}\right)\right) \geq 0 \\ -1 & \text { otherwise }\end{cases}
$$

where $Q\left(h_{s}^{k}\right)$ is the $h_{s}^{k}$ th element of $Q$ in zigzag order. Also we define two cases:

$$
\begin{aligned}
& \text { case1: } \mathscr{W}_{\mathrm{L}}\left(\operatorname{Index}\left(h_{\mathrm{s}}^{\mathrm{k}}\right)\right)=\operatorname{LSB}\left(\left|R C_{i, j}\left(h_{\mathrm{s}}^{k}\right)\right|\right) \\
& \text { case2: } \mathbb{W}_{\mathrm{M}}\left(\operatorname{Index}\left(h_{\mathrm{s}}^{k}\right)\right)=\operatorname{LSB}\left(\left|R C_{i, j}\left(h_{\mathrm{s}}^{k}\right)\right|\right)
\end{aligned}
$$

here $\operatorname{Index}(\cdot)$ means the corresponding index of watermark vector and $\mathrm{LSB}(\cdot)$ denotes the least significant bit. The embedding strategy can be expressed as:

(a) For $s=\{1,2\}$, embbeding $\mathbb{V}_{L}$,

$$
C_{i, j}\left(h_{s}^{k}\right)= \begin{cases}R C_{i, j}\left(h_{s}^{k}\right) \times Q\left(h_{s}^{k}\right) & \text { if casel } \\ \left(R C_{i, j}\left(h_{s}^{k}\right)+S g n\right) \times Q & \left(h_{s}^{k}\right) \text { else }\end{cases}
$$

(b) For $s=\{3,4\}$, embbeding $\mathbb{W}_{M}$,

$$
C_{i, j}\left(h_{s}^{k}\right)= \begin{cases}R C_{i, j}\left(h_{s}^{k}\right) \times Q\left(h_{s}^{k}\right) & \text { if case2 } \\ \left(R C_{i, j}\left(h_{s}^{k}\right)+S g n\right) \times Q & \left(h_{s}^{k}\right) \text { else }\end{cases}
$$

After all the blocks of the coefficient matrix $C$ are watermarked, performing IDCT to the watermarked coefficient matrix and we get the watermarked image ${ }^{\mathrm{W}}$.

\section{Procedure of Authentication}

The procedure of authentication can be performed without the reference of original image, thus this algorithm is obliviousness. Assume $I^{*}$ is the watermarked image under attack at the receiver end. The authentication scheme can be describe as follows:

- Firstly, processing the image $\mathrm{I}^{*}$ with the same steps performed in the feature extracting, and get the watermark vectors $\mathscr{V}_{L}^{*}, W_{M}^{*}$ from block pairs. Also, extracting watermarking $\mathscr{W}_{H}^{*}$ from high frequency domain in each block. Verifying the embedded coefficients of block $C_{i, j}^{*}$ as follows:

(a) For $s=\{1,2\}$,

$\operatorname{Ver}\left(C_{i, j}^{*}\left(h_{s}^{k}\right)\right)=\left\{\begin{array}{l}0 \text { if } \mathbb{F}_{L}^{*}\left(I n d e x\left(h_{s}^{k}\right)\right)=\mathbb{F}_{H}^{*}\left(I n \operatorname{dex}\left(h_{s}^{k}\right)\right) \\ 1 \text { else }\end{array}\right.$

(b) For $s=\{3,4\}$,

$\operatorname{Ver}\left(C_{i, j}^{*}\left(h_{s}^{k}\right)\right)=\left\{\begin{array}{l}0 \text { if } \mathbb{P}_{M}^{*}\left(I n \operatorname{dex}\left(h_{s}^{k}\right)\right)=\mathbb{P C}_{H}^{*}\left(\operatorname{Index}\left(h_{s}^{k}\right)\right) \\ 1 \text { else }\end{array}\right.$

- Then the integrity of each block can be authenticated with the following rule (9), and $\mathrm{C}_{\mathrm{i}, \mathrm{j}}^{*}$ will be marked as "invalid" if Auth $\left(C_{i, j}^{*}\right) \geq 3$, otherwise marked as "valid". The authentication result of this scheme will be show in the latter section. 


$$
\operatorname{Auth}\left(C_{i, j}^{*}\right)=\sum_{s=1}^{4} \operatorname{Ver}\left(C_{i, j}^{*}\left(h_{s}^{k}\right)\right)
$$

\section{CUDA-BASED IMPLEMENTATION}

\section{A. D etails of Watermarked Image Generation with CUDA}

Looking into the image authentication algorithm proposed in section IV, we can find that the amount of calculation of the algorithm centralized on the process of DCT and watermark extracting and embedding, which are highly amenable to parallel processing. Thus parallelizing these processing will accelerate the performance of the algorithm considerably. The CUDAbased watermarked image generation will be implemented with a CUDA kernel function named CuAIG-Kernel, as illustrated in Fig.3.

The CuAIG-Kernel is implemented on a grid of $\mathrm{M} / 32 \times \mathrm{N} / 32$ thread blocks, and each block consists of $8 \times 4 \times 4$ threads. The implementation of CuAIG-Kernel is described below.

For computing partition, we firstly further divided the DCT coefficient Matrix C into $4 \times 4$ non-overlapped macro-blocks, namely each macro-block consists of sixteen $8 \times 8$ blocks, as showed in Fig. 4 .

In the executing of DCT, we decompose the task into series of macro-blocks, and they are mapped into equal numbers of CUDA blocks, that is one thread block is

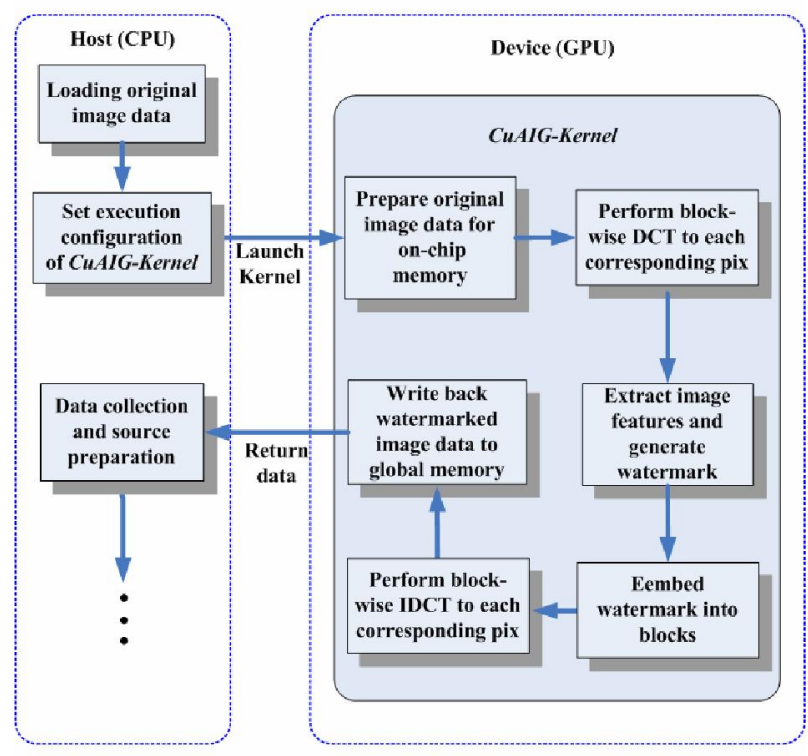

Figure 3. Executing process of CUDA-based watermarked image generation

allocated to process its corresponding pixel of macroblock. Because of shared memory (on-chip memory) has lower latency and much higher bandwidth than global memory, the data accessed by each thread block will be read firstly from the global memory to shared memory before doing any arithmetic. As illustrated by Fig.5, each thread reads 8 element (one column) of $8 \times 8$ block into shared memory, and computes the corresponding DCTcoefficients for each $8 \times 8$ block, thus a warp of threads will round off the DCT-coefficients' computing of four
$8 \times 8$ blocks. As the equation (10) showed, the performing of $2 \mathrm{D}-\mathrm{DCT}$ on sample $X$ can subsequently

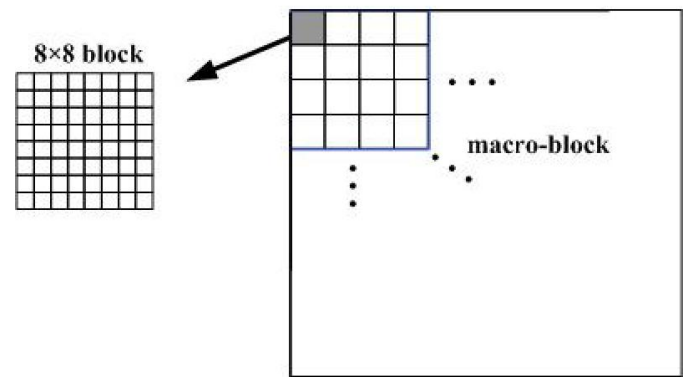

Figure 4. Divide block-wise matrix into non-overlapped macro-blocks

apply DCT to columns $\left(Y=A^{\top} X\right)$ and rows $(C=Y A)$ of the input signal:

$$
C(u, v)=\left(A^{\top} X\right) A
$$

where $A$ is an 8 -order cosine matrix, $X$ is a $8 \times 8$ block. To make the DCT-computing of each block even more efficiently, we also employed the high redundancy and symmetry of the elements of matrix A. More detail can be seen in [11].

While performing the step of watermark extracting, 8 threads as showed in Fig.6 (a) involved to generate watermark vectors $W_{L}$ and $W_{M}$ by the rule defined in section IV.

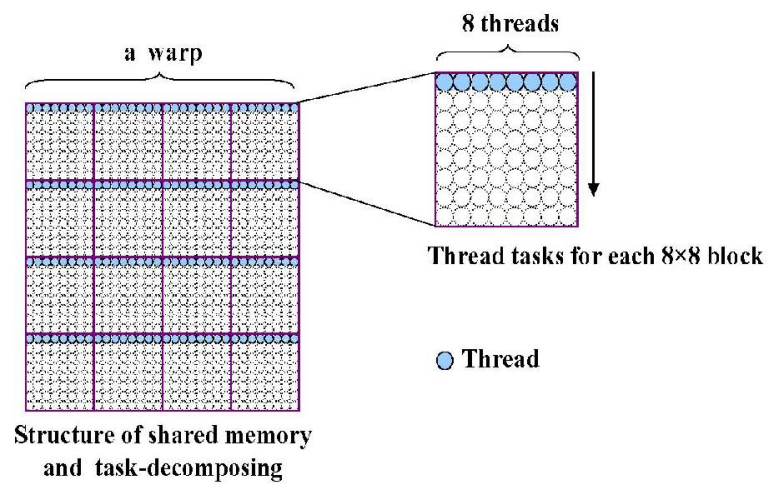

Figure 5. Structure of shared memory and thread-tasks decomposing in each thread block of CUDA

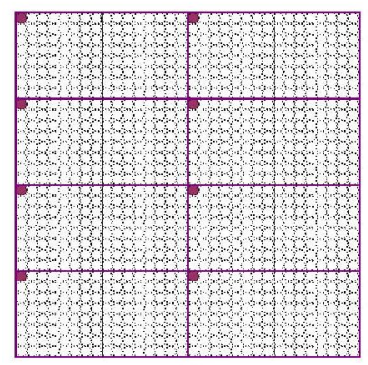

(a)

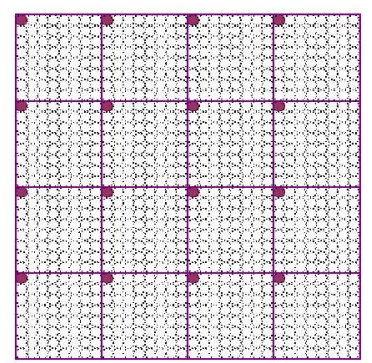

(b)
Figure 6. (a) 8 threads involved in watermark extracting; (b) 16 threads involved in watermark embedding

In the step of embedding watermark, 16 threads will be involved to embed several elements of the previous 
watermark with the rules of (6) (7), as illustrated by Fig. 6 (b). After all watermark elements are embedded, all threads of each CUDA block are involved in performing inverse DCT (IDCT) to the block-wise coefficients and got the final watermarked image data.

At last, all threads are involved to write all of the DCTcoefficients back to global memory with the inverse procedure of reading.

\section{EXPERIMENTAL RESULTS}

In this section, we will present the experiment results of the proposed algorithm for image authentication on both CPU and GPUs. As for the CPU implementation, the algorithm is implemented by using primary-optimized single-threaded $\mathrm{C}$ language. Both $\mathrm{CPU}$ and CUDA implementations of the algorithm have been performed under a workstation computer equipped with two NVIDIA Tesla C1060 devices. The host's parameters are showed in Table I and Table II shows the main properties of NVIDIA Tesla C1060.

TABLE I.

MAIN CONFIGURATION PARAMETERS OF TEST ENVIRONMENT

\begin{tabular}{l|l}
\hline Parameters & Values \\
\hline CPU & Intel Xeon 5520 $(2.26 \mathrm{GHz})$ \\
RAM & 12GB DDR3 $(1333 \mathrm{MHz})$ \\
GPU Architecture & Tesla C1060 \\
OS & Centos $5.3(64 \mathrm{bit})$ \\
CUDA & V2.3 \\
\hline
\end{tabular}

TABLE II

PROPERTIES OF NVIDIA TESLA C 1060

\begin{tabular}{l|l}
\hline Parameter s & Values \\
\hline Number of multiprocessors & 30 \\
Number of cores & 240 \\
Global Memory & $4 \mathrm{~GB}$ \\
Shared Memory per block & $16 \mathrm{~KB}$ \\
DRAM & $4 \mathrm{~GB}$ \\
Registers per block & 16384 \\
Clock Rate & $1.3 \mathrm{GHz}$ \\
\hline
\end{tabular}

\section{A. Results of Authentication}

In the experiment of image authentication, we choose three BMP format image (Lena, Pepper, and Baboon) to demonstrate the effects of the proposed algorithm both on CPU and GPU.

\section{a) Imperceptibility}

Fig.7 shows the original images and the results of watermarked images on CPU and CUDA version, which fit the imperceptibility as we expected. Table III displays the average PSNR (db) of CPU-processed and GPUprocessed Images $(512 \times 512$ size $)$, and also compare with Lin's related work [13]. Fig.8 shows the PSNR values of GPU-processed Images in varied sizes.

\section{b) Localization of tampering}

Fig.9 shows the detected results of the tamperedimages $(512 \times 512$ size) using CUDA version, from which we can see the accurately locating of tampered regions with the proposed algorithm.

\section{c) Tolerance of JPEG compression}

Fig.10 illustrates the watermarked image undergoing different scales of JPEG compression and their respective authentication results. When the compression scales under the pre-quantization value, authentication results come out be resisting the compression and stay integrity with images before authentication.

\section{B. Performance of CUDA-based Parallelization}

To illustrate the accelerating performance of CUDA, we compare the executing time of the proposed CUDA method with CPU version, both executing time of CPU and CUDA version are average values of experiment results in 10 times' running. The speedups achieved with the proposed CUDA implementation range from $20 \mathrm{x}$ $(512 \times 512$ size $)$ to $50 x(8192 \times 8192$ size $)$, as illustrate by Fig.11.

To make full use of the computing resource of GPU devices, the algorithm was also implemented on multiple GPUs. Two NVIDIA Tesla C1060 devices are involved in the batch processing of images $(1024 \times 1024$ size $)$.

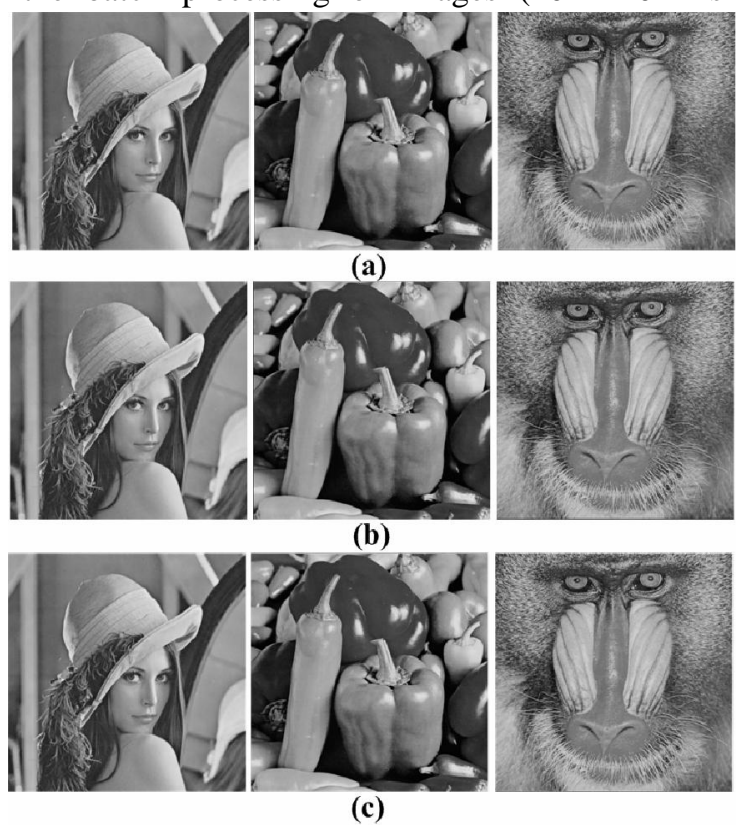

Figure 7. (a) Original images; (b) Watermarked images generated by CPU version; (c) Watermarked images generated by CUDA version

TABLE III

PSNR OF CPU-PROCESSED AND CUDA-PROCESSED IMAGES

\begin{tabular}{c|ccc}
\hline & Lena & Pepper & Baboon \\
\hline CPU & 39.26 & 39.36 & 38.77 \\
GPU & 39.24 & 39.26 & 38.65 \\
Lin[12] & 37.38 & 36.97 & 40.52 \\
\hline
\end{tabular}

Table IV shows the executing time of watermarked processing on CPU, single GPU and dual GPU with different amount of files, and we can see that the system gains higher work efficiency with two GPUs than single GPU.

\section{CONCLUSIONS AND FUTURE WORK}

An image content authentication algorithm is presented and its CUDA-based parallel implementation is also presented in this paper. Experimental results have shown 
that the performance of the scheme is effective both in authentication results and operations speedup. And further more, multiple GPUs implementation are also employed to make full used of the GPU devices, which works $1.2 \mathrm{x}$ times faster than single GPU method. The study of this paper is mainly focuses on acceleration of the image authentication scheme with a novel CUDAbased manner. It is truly that the proposed authentication algorithm can be improved to make it more robust to unintentional manipulation and even be able to recovery the tamper region. Future work involves a more in depth analysis of the optimizations and parallelization strategy of CUDA program in order to further exploit the high performance of GPU's computation capability, and also extending the CUDA-based method to authentication method within wavelet transform domain.

\section{ACKNOWLEDGMENT}

The paper is supported by National Natural Science Foundation of China (No. 61073061). The authors are grateful to all the people for helpful suggestions. The authors would like to thank all the anonymous reviewers for their helpful comments on earlier drafts of this paper.

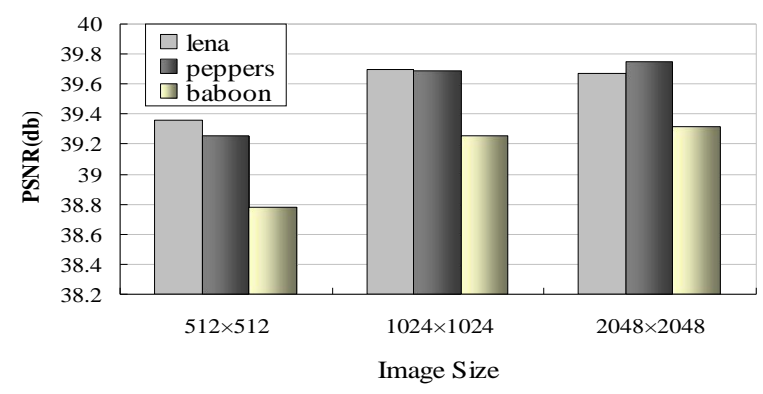

Figure 8. PSNR of GPU-processed images in varied sizes
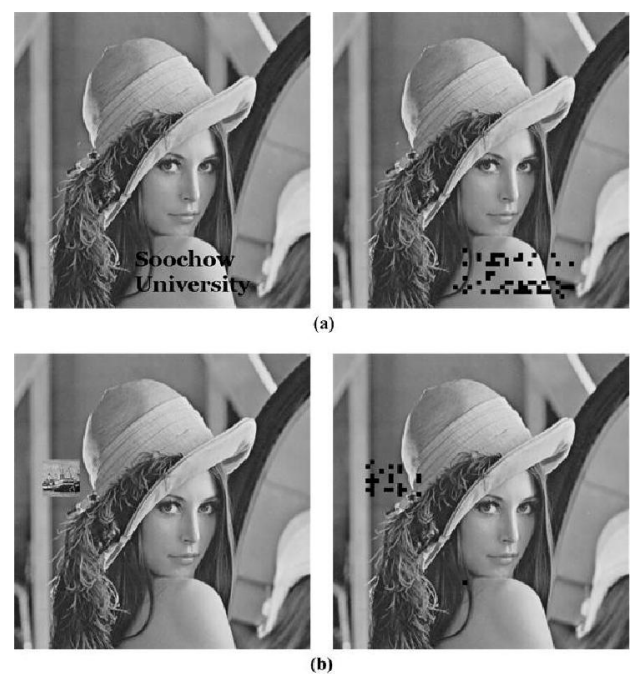

Figure 9. (a) Modification-attacked image and detected result by CUDA version; (b) Collage-attacked image and detected result by CUDA version

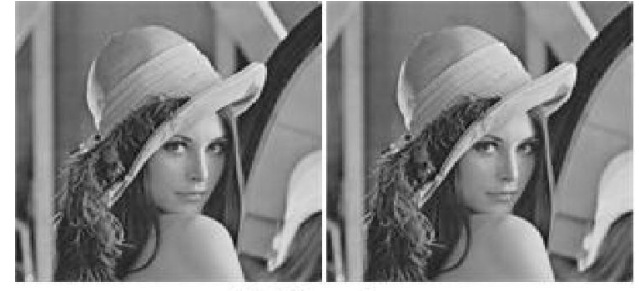

(a) Q/8 scale compress

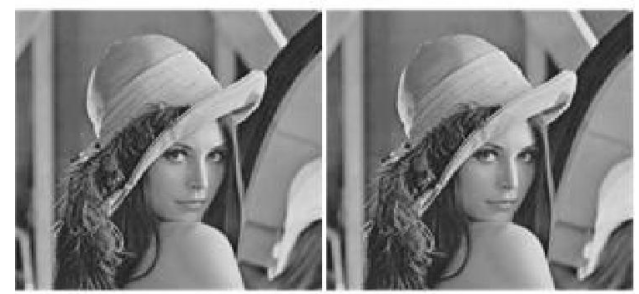

(b) Q/6 scale compress

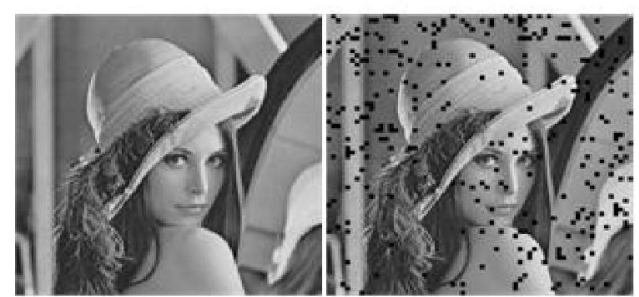

(c) $Q$ scale compress

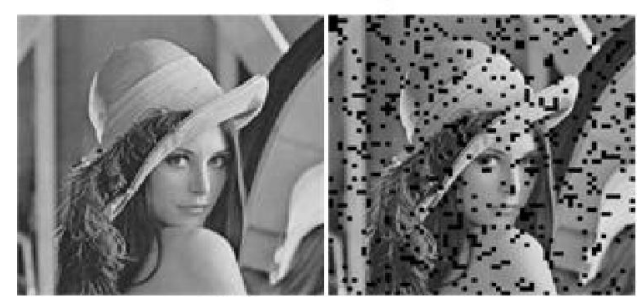

(d) $2 \mathrm{Q}$ seale compress

Figure 10. Different scale of JPEG compression of watermarked images and respective authentication results

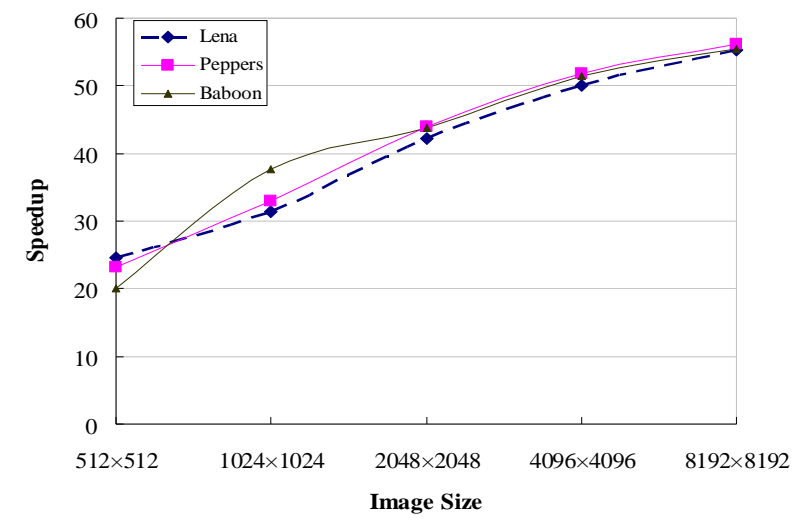

Figure 11. Speedup of the generation of watermarked images on GPU compared with CPU version

TABLE IV

EXECUTING TIME OF DIFFERENT NUMBERS OF IMAGES

\begin{tabular}{c|ccc|c}
\hline \multirow{2}{*}{$\begin{array}{c}\text { Computing } \\
\text { device }\end{array}$} & \multicolumn{3}{|c|}{ Executing time $(\mathrm{msec})$} & \multirow{2}{*}{$\begin{array}{c}\text { Speedup } \\
\text { vs. CPU }\end{array}$} \\
\cline { 2 - 3 } & \multicolumn{2}{|c|}{ Number of Images $(1024 \times 1024$ size $)$} & \multirow{2}{*}{2000} & \\
\hline CPU & 7153.62 & 14291.22 & 28877.66 & -- \\
Single GPU & 220.53 & 440.85 & 903.83 & $32 \mathrm{x}$ \\
Dual GPU & 179.37 & 378.60 & 646.27 & $40 \mathrm{x}$ \\
\hline
\end{tabular}




\section{REFERENCES}

[1] A. Haouzia and R. Noumeir, "Methods for image authentication: a survey," Multimedia Tools Appl, pp. 1 46, 2008.

[2] T. Chen, J. C. Wang, and Y. L. Zhou, "Combined digital signature and digital watermark scheme for image authentication," International Conferences on Info-tech and Info-net, Vol. 5, pp. 78 - 82, 2001.

[3] C. K. Ho and C. T. Li, "Semi-fragile watermarking scheme for authentication of jpeg images," In Information Technology: Coding and Computing, 2004, Proceedings, Vol. 1, pp. 7 - 11, 2004.

[4] C. T. Li, "Digital fragile watermarking scheme for authentication of jpeg images," IEE Proc.-Vis. Image Signal Process., Vol. 151, pp. 460 - 466, 2004.

[5] C. Y. Lin and S. F. Chang, "A robust image authentication method distinguishing jpeg compression from malicious manipulation," Circuits and Systems for Video Technology, IEEE Transactions on, Vol.11, pp.153-168, 2001.

[6] E. Kougianos, S. P. Mohanty, and R. N. Mahapatra, "Hardware assisted watermarking for multimedia," Computers Electrical Engineering, Vol.35, pp. 339 - 358, 2009.

[7] A. Brunton and J. Y. Zhao, "Real-time video watermarking on programmable graphics hardware," In Electrical and Computer Engineering, 2005. Canadian Confer ence on, pp. $1312-1315,2005$.

[8] S. P. Mohanty, N. Pati, and E. Kougianos, "A watermarking co-processor for new generation graphics processing units," In Consumer Electronics, 2007. Digest of Technical Papers. International Conference on, pp. 1-2, 2007.

[9] NVIDIA Corporation, "NVIDIA CUDA Programming Guide_Version1.1,"http://developer.download.nvidia.com/ compute/cuda/1_1/NVIDIA_CUDA_Programming_Guide 11.pdf, 2007.

[10] H. Kourkchi and S. Ghaemmaghami, "Improvement to a semi-fragile watermarking scheme against a proposed counterfeiting attack," Advanced Communication Technology, 2009. 11th International Conference on, Vol. 03, pp. 1928-1932, 2009

[11] C. Y. Lin and S. F. Chang, "Sari: self-authentication-and recovery image watermarking system," in MULTIMEDIA '01: Proceedings of the ninth ACM international conference on Multimedia, pp. 628 - 629, 2001.

[12] A. Obukhov and A. Kharlamov, "Discrete Cosine Transform for $8 \times 8$ Blocks with CUDA," NVIDIA white paper, 2008.

[13] C. H. Lin, T. S. Su and W. S. Hsieh, "Semi-Fragile Watermarking Scheme for Authentication of JPEG
Images," Tamkang Journal of Science and Engineering, Vol. 10(1), pp. 57-66, 2007.

[14] S. P. Mohanty, E. Kougianos and N. Ranganathan, "VLSI architecture and chip for combined invisible robust and fragile watermarking," Computers Digital Techniques, IET, Vol. 1(5), pp. 600-611, 2007.

[15] Y. H. Seo and D.W. Kim, "Real-time blind watermarking algorithm and its hardware implementation for motion JPEG2000 image codec," In Proceedings of the 1st workshop on embedded systems for real-time multimedia, pp. 88-93, 2003

[16] S. Lahabar, P. J. Narayanan, "Singular value decomposition on GPU using CUDA," IEEE International Symposium on Parallel \& Distributed Processing, pp. 1-10, 2009.

[17] S. H. Yoo, J. H. Park, C. S. Jeong, "Accelerating Multiscale Image Fusion Algorithms Using CUDA," International Conference of Soft Computing and Pattern Recognition, SOCPAR '09, pp. 278-282, 2009.

[18] S. Chen, J. Qin, Y. M. Xie, W. M. Pang, P. A. Heng, "CUDA-based acceleration and algorithm refinement for volume image registration," International Conference on Future Biomedical Information Engineering, FBIE 2009, pp. 544-547, 2009.

[19] S. Datla, N. S. Gidijala, "Parallelizing Motion JPEG 2000 with CUDA," Computer and Electrical Engineering, ICCEE '09. Second International Conference on, pp. 630634, 2009.

Caiwe L in received the B.S. degree in 2008 from Yangzhou University, Yangzhou, China. He is now a M.S. candidate in the school of computer science and technology of Soochow University. His current research areas are digital image processing, parallel and distributed computing.

Lei Zhao received the Ph.D. degree in 2006 from Soochow University, Suzhou, China. He has been a faculty member of the school of computer science and technology of Soochow University since 1998. He is now Associate Professor at the Department of Network Engineering. His research interests include distributed data processing, data mining, parallel and distributed computing.

J iwen Y ang received the B.S. degree in 1984 from Nanjing Normal University, Nanjing, China. He has been a faculty member of the school of computer science and technology of Soochow University since 1984. He is now Professor at the Department of Information Management. His research interests include distributed data processing, management information system, parallel and distributed computing. 